\title{
La miel como alternativa a los tratamientos tópicos en el proceso de curación de quemaduras, heridas y úlceras
}

\section{Honey as an alternative to topical treatments in the healing process of burns, wounds and ulcers}

\author{
SaraGarcía Felipe ${ }^{(1)}$ \\ 1. Enfermera en Centro de Mayores Casablanca.
}

Recibido: 30 de julio de 2018

Aceptado: 05 de abril de 2019

\section{Resumen:}

Introducción: Las lesiones por quemaduras, heridas quirúrgicas y úlceras causan altos niveles de morbilidad y mortalidad en todo el mundo, siendo particularmente vulnerables a infecciones. Diversos estudios sugieren que la miel puede acelerar la curación de heridas debido a sus propiedades, como su capacidad para reducir la inflamación y prevenir el ataque de agentes infecciosos. Objetivo: Valorar la evidencia científica sobre la efectividad de la miel como un tratamiento alternativo en quemaduras, heridas o úlceras. Material y método: Se realizó una búsqueda bibliográfica en bases de datos especializadas como Pubmed, Cochrane, Scopus y WoS entre otras. Se encontraron un total de 11.303 artículos que fueron cribados mediante criterios de inclusion y exclusion. Fueron incluidos 26 (19 ECAs y 7 revisiones 
sistemáticas), los cuales fueron evaluados para valorar la calidad y el riesgo de sesgo mediante las escalas PEDro y AMSTAR, y posteriormente por la escala SIGN.

Resultados: Se observó que la miel cicatriza antes y deja menos cicatriz hipertrófica, reduce el edema, el dolor y las secreciones purulentas, produce una mayor esterilidad en menos tiempo y con menos efectos adversos además de ser más costo-efectiva.

Conclusiones: La miel se puede utilizar para curar este tipo de lesiones suponiendo una alternativa de tratamiento pudiendo mejorar la asistencia sanitaria.

Palabras clave: miel; herida; quemadura; úlcera

\begin{abstract}
:
Introduction: Injuries from burns, surgical wounds and ulcers cause high levels of morbidity and mortality throughout the world, being especially vulnerable to infections. Several studies suggest that honey can accelerate the healing of wounds due to its properties, as its ability to reduce inflammation and prevent the attack of infectious agents.
\end{abstract}

Objective: to assess the scientific evidence on the effectiveness of honey as an effective alternative treatment in burns, wounds or ulcers.

Material and method: A literature search was carried out in specialized databases such as Pubmed, Cochrane, Scopus and WoS, among others. A total of 11,303 articles were found that were screened by inclusion and exclusion criteria. We included 26 (19 RCTs and 7 systematic reviews), which were evaluated to assess the quality and risk of bias using the PEDro and AMSTAR scales, and subsequently by the SIGN scale.

Results: It was observed that honey heals before and leaves less hypertrophic scar, reduces edema, pain and purulent secretions, produces greater sterility in less time and with less adverse effects besides being more cost-effective. 
Key Words: honey; wound; burn; ulcer.

\section{Introducción}

En la mayoría de los países, las quemaduras son una causa importante de muerte accidental, así como de pérdida de años de vida ajustados en función de la discapacidad, tan sólo superado por los accidentes de automóviles $\left.{ }^{(1}, \underline{2}\right)$.

La etiología de las quemaduras puede agruparse en varias categorías: por líquidos calientes, metales calientes, fuego, elementos eléctricos, químicos o radiación, siendo las escaldaduras por líquidos calientes las responsables del $85 \%$ de todas las lesiones por quemaduras. En cuanto a las relacionadas con incendios representan alrededor de 10 millones de discapacidades, incluyendo personas con contracturas de la herida y otras deficiencias físicas que limitan sus capacidades funcionales, así como la posibilidad de llevar una vida normal y económicamente productiva. Sin embargo, excluye los impactos de las desfiguraciones que a menudo van asociadas a un estigma social $(1,2,3)$.

A pesar de las medidas profilácticas, los últimos datos encontrados en España, en 2011, estiman que 3 de cada 1.000 habitantes sufren cada año quemaduras que requieren atención médica, siendo la mayoría atendidos en Atención Primaria (AP), y entre el $15-20 \%$ requieren ingreso hospitalario (4).

Por otro lado, se considera que la aparición de infecciones en heridas quirúrgicas constituyen el segundo grupo más numeroso de infecciones intrahospitalarias (después de las urinarias) duplicando la estancia y los costes hospitalarios. Según el análisis EPINE-EPPS de 2016 del Ministerio de Sanidad y Consumo en España, las IHQ cifran un $21,6 \%$ del total de infecciones nosocomiales, prolongando su estancia hospitalaria entre 8 y 10 días y aumentando unos $1.660 €$ los gastos por paciente $(5,6)$.

En cuanto a las úlceras, la prevalencia en España en 2013 de UPP nosocomiales suponen un $65,6 \%$ del total, siendo la prevalencia más alta en la unidad de cuidados intensivos ( $\mathrm{UCl}$ ), llegando al $18 \%{ }^{(8,9)}$. Respecto a otro tipo 
de ulceras, la incidencia de úlcera neuropática es de un $46 \%$, la isquémica de un $12 \%$ y las úlceras de pie diabético un $25 \%$ (el $85 \%$ de éstas terminan en amputación) (10).

Una de las variables que los estudios señalan es el importante impacto de estas lesiones centrado en los cambios de la imagen corporal, en la que tiene gran importancia las cicatrices, las cuales producen importantes alteraciones emocionales, dificultan los procesos adaptativos, presentando frecuentemente conductas y/o actitudes de evitación social tales como evitar salir del hogar, exponer las cicatrices a otras personas y evitar relaciones interpersonales 0 sexuales. Este comportamiento es debido al "rechazo social" que perciben cuando los otros les miran fijamente, les preguntan sobre el origen de las cicatrices, realizan comentarios, etc ${ }^{(11,12,13)}$.

En relación al tratamiento tópico de estas heridas es frecuente aplicar pomadas que tienen una función antibiótica de amplio espectro. La pomada de aplicación tópica más utilizada es la crema estéril de sulfadiazina argéntica al 1 $\%$ (Flammazine $\AA$, Silvederma $\AA$ ), seguida de la pomada con nitrofurazona $0.2 \%$ (Furacín®) y los geles de povidona yodada (Betadine Gel®). Otros tratamientos son hidrogeles, espumas, alginatos, hidrofibras de hidrocoloide, apósitos de plata, y otros que mantengan un ambiente aséptico y que favorezca la cicatrización $(\underline{14,15})$.

Entre los tratamientos menos convencionales se encuentra la miel, solución azucarada viscosa que contiene fundamentalmente un $75-79 \%$ de azúcar y un $20 \%$ de agua. Los azúcares mayoritarios son glucosa $30 \%$ y fructosa $40 \%$ y en menos cantidad sacarosa, maltosa y otros azúcares complejos. Otros componentes son las proteínas, vitaminas del complejo B y $\mathrm{C}$, minerales, sustancias inhibidoras de gérmenes (germicida e inhibina), antioxidantes (flavonoides, ácido ascórbico y selenio), ácidos orgánicos (siendo el ácido Glucurónico el más abundante) responsables de su pH bajo entre 3.5 y 6 aproximadamente; así como enzimas (invertasa, diastasa, catalasa y glucosa oxidasa), que juegan un papel importante en las propiedades curativas de la miel (16-18). Dentro de sus efectos se encuentran los siguientes: 
Efecto antimicrobiano: incluso cuando la miel se diluye, la acción antibacteriana es 1.000 veces más concentrada que los antisépticos de uso común debido su alto contenido en azúcar y su efecto osmótico que dificultan el crecimiento microbiano, por lo tanto promueve la curación sin efectos adversos (17-21). Asimismo, la miel puede ser efectiva contra las cepas resistentes a los antibióticos (17, 19).

Desbridamiento autolítico y desodorización: La rápida desodorización de las heridas se debe a que la miel es una fuente rica en glucosa que las bacterias metabolizan produciendo ácido láctico (metabolito no oloroso) (20,21).

Por otra parte, el fácil desbridamiento autolítico es debido a su acción osmótica, que disminuye el edema creando un ambiente húmedo mediante la extracción de líquido linfático de los tejidos (19-21).

Actividad antiinflamatoria y antioxidante: El mecanismo por el cual se reduce la inflamación está relacionado con los antioxidantes de la miel los cuales limpian los radicales libres producidos en la etapa inflamatoria, disminuyendo el estrés oxidativo y por tanto el proceso inflamatorio. Además, la miel reduce los niveles de prostaglandinas (principal sustancia favorecedora de la inflamación), que junto con la eliminación de bacterias infecciosas que estimulan la respuesta infamatoria, produce una disminución de la inflamación (ㅂ-21).

Actividad estimulante del crecimiento del tejido de granulación, epitelización y angiogénesis: El efecto cicatrizante de la miel es debido al resultado combinado de la creación de un ambiente húmedo (favoreciendo la multiplicación celular), del desbridamiento del tejido necrótico y desvitalizado, la absorción del edema, la promoción de la granulación y epitelización y sus propiedades bactericidas y fungicidas. Además, su acidez provoca una mayor oxigenación de la sangre, lo que favorece la reparación tisular (17-21).

Propiedades antivirales: Solo existe en la actualidad un ensayo sobre el uso de miel en lesiones herpéticas (labiales y genitales) que mostró que la aplicación tópica de miel tuvo mejores resultados, sin efectos secundarios y fue efectivo en el manejo del dolor y otros signos y síntomas. Sin embargo, hay una 
escasez de investigación con respecto a las propiedades antivirales de la miel (17).

Debido a que estas propiedades pueden favorecer el proceso de curación, nace el presente trabajo con la finalidad de evidenciar la efectividad de la miel como tratamiento alternativo en pacientes que sufren quemaduras, heridas 0 úlceras en comparación con los tratamientos tópicos convencionales.

\section{Objetivo: pregunta PICO}

El objetivo de esta revisión es valorar la evidencia científica sobre la efectividad de la miel como un tratamiento alternativo en quemaduras, heridas o úlceras.

\section{PREGUNTA PICO}

- P: Pacientes que sufren quemaduras, heridas o ulceras en alguna zona de su cuerpo.

- I: Aplicación de tratamiento natural, la miel

- C: Aplicación de tratamientos tópicos

- O: El tratamiento natural de la miel cura antes y presenta más beneficios que los tratamientos de uso tópico.

- S: Ensayos Clínicos Aleatorizados (ECAS) y revisiones sistemáticas.

\section{Material y Método}




\section{Criterios de inclusión de los estudios.}

Tabla 1. Criterios de inclusión.

\begin{tabular}{|l|l|}
\hline Criterios de inclusión & Criterios de exclusión \\
\hline Artículos Full text & Estudios realizados con animales \\
\hline Fecha: $<10$ años & Idioma distinto de inglés/español \\
\hline Artículos de acceso gratuito & Edad: neonatos o niños < 3 años \\
\hline Idioma (ingles/español) & Tratamiento que no fuera tópico \\
\hline $\begin{array}{l}\text { Estudios con muestra poblacional en el rango } \\
\text { de } 3 \text { a } 80 \text { años. }\end{array}$ & \\
\hline
\end{tabular}

\section{Fuentes de información (bases de datos).}

Los estudios se identificaron mediante búsquedas electrónicas en bases de datos bibliográficas. Se eligieron aquellas en español y/o inglés. Se consultaron las siguientes bases de datos:

- Scopus

- Web of Science (WoS)

- Csic: IME

- Tripdatabase

- Cochrane

- Pubmed

- Google académico

- SCiELO

- Elservier 
- PEDro

- EUROPE PMC

- MEDES

El período de búsqueda de la bibliografía se inició en el mes de octubre de 2017 finalizando en el mes de mayo de 2018. La última búsqueda se realizó el 10 de mayo de 2018.

\section{Estrategia de búsqueda (combinación de términos de búsqueda).}

Se realizó una búsqueda bibliográfica utilizando la terminología MeSH y DeCS mediante las palabras clave "honey", "wound", "burn”, "ulcer", "blind" y "controlled clinical trial" y se emplearon operadores booleanos siendo la cadena de búsqueda inicial "honey AND burn OR wound OR ulcer AND blind OR controlled clinical trial". A continuación se añadieron truncamientos y paréntesis.

La cadena de búsqueda resultante en la búsqueda de la información fue la misma en todas las bases de datos y está compuesta por los siguientes términos:

Honey AND (wound* OR burn* OR ulcer*) AND (randomized OR "random allocation" OR RCT* OR "controlled clinical trial" OR "double blind" OR "doubleblind" OR "single blind" OR "single-blind" OR nonblinded)

\section{Selección de los estudios y recolección de datos.}

Se identificaron los estudios potencialmente incluibles a partir del título y del resumen. Posteriormente se analizó el texto completo para su selección y se 
cribaron mediante los criterios de inclusión y exclusión. Finalmente se incluyeron 26 artículos (Figura 1 ).

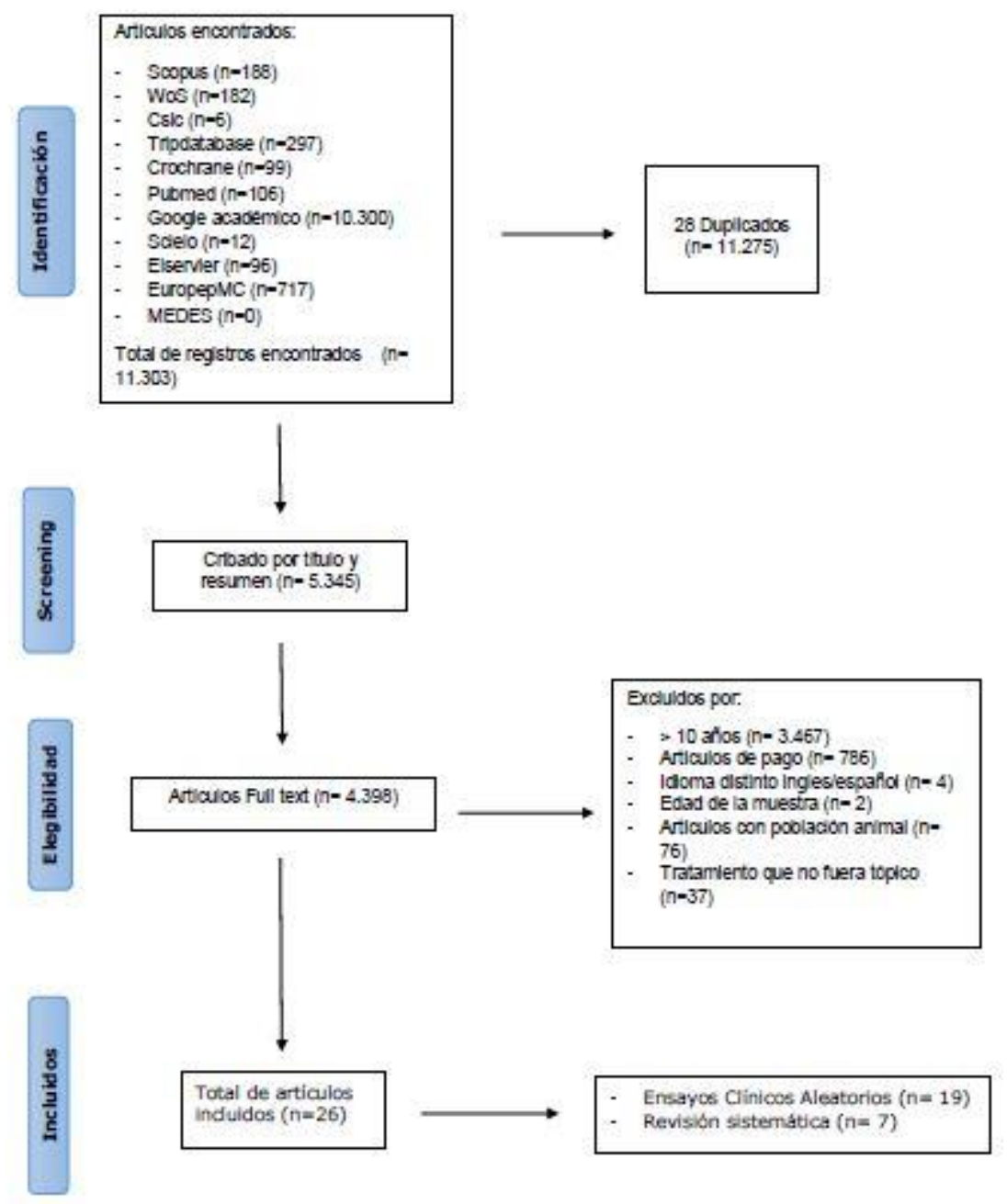

Figura 1. Diagrama de flujo. Selección de estudios.

\section{Evaluación de la calidad de los estudios: Detección de posibles sesgos.}

Para evaluar el riesgo de sesgo en los estudios se utilizaron distintas escalas, para los Ensayos Clínicos Aleatorizados (ECAS) se utilizó la escala PEDro y para las revisiones sistemáticas se utilizó la escala AMSTAR. 
La puntuación de la calidad metodológica osciló entre 5 y 10 sobre 11 ítems (media $=7,5$ ) según la escala PEDro y 4 y 9 sobre 11 ítems (media $=6,1$ ) según la escala AMSTAR.

Tabla 2. Resultados de la escala PEDro

\begin{tabular}{|c|c|c|c|c|c|c|c|c|c|c|c|c|}
\hline Autores & 1 & 2 & 3 & 4 & 5 & 6 & 7 & 8 & 9 & 10 & 11 & Puntuación \\
\hline $\begin{array}{l}\text { Baghel et al. } \\
2009\end{array}$ & $S$ & $\mathrm{~s}$ & $\mathrm{~N}$ & S & $\mathrm{N}$ & N & $\mathrm{N}$ & $\mathrm{s}$ & $S$ & $S$ & $S$ & 7 (bueno) \\
\hline $\begin{array}{l}\text { Yapucu Günes U } \\
\text { et al. } 2007\end{array}$ & $N$ & $S$ & $\mathrm{~N}$ & $\mathrm{~s}$ & $\mathrm{~N}$ & $\mathrm{~N}$ & $\mathrm{~N}$ & $\mathrm{~s}$ & $S$ & S & $\mathrm{N}$ & 5 (justo) \\
\hline $\begin{array}{l}\text { Kamaratos et al. } \\
2014\end{array}$ & $S$ & $\mathrm{~s}$ & $\mathrm{~N}$ & $\mathrm{~s}$ & $\mathrm{~s}$ & $S$ & $\mathrm{~N}$ & $\mathrm{~s}$ & $\mathrm{~s}$ & $\mathrm{~s}$ & $\mathrm{~s}$ & $\begin{array}{l}9 \text { (muy } \\
\text { bueno) }\end{array}$ \\
\hline Singh et al. 2011 & $S$ & S & $\mathrm{N}$ & $\mathrm{s}$ & $\mathrm{N}$ & $\mathrm{N}$ & $\mathrm{N}$ & $\mathrm{S}$ & $S$ & S & $S$ & 7 (bueno) \\
\hline Ingle et al. 2006 & $S$ & $\mathrm{~s}$ & $\mathrm{~N}$ & $\mathrm{~s}$ & $\mathrm{~s}$ & $S$ & $\mathrm{~N}$ & $\mathrm{~S}$ & $\mathrm{~S}$ & $\mathrm{~s}$ & $\mathrm{~s}$ & $\begin{array}{l}9 \text { (muy } \\
\text { bueno) }\end{array}$ \\
\hline $\begin{array}{l}\text { Saeidinia et al. } \\
2017\end{array}$ & $S$ & $S$ & $\mathrm{~N}$ & $\mathrm{~s}$ & $S$ & $S$ & $\mathrm{~N}$ & $\mathrm{~s}$ & $\mathrm{~s}$ & $\mathrm{~S}$ & $\mathrm{~s}$ & $\begin{array}{l}9 \text { (muy } \\
\text { bueno) }\end{array}$ \\
\hline Imran et al. 2015 & $S$ & $S$ & $\mathrm{~N}$ & S & S & $S$ & $\mathrm{~N}$ & $\mathrm{~N}$ & $\mathrm{~S}$ & S & $\mathrm{N}$ & 7 (bueno) \\
\hline Nurus et al. 2011 & $S$ & $S$ & $\mathrm{~N}$ & S & N & N & N & $S$ & $S$ & $S$ & $S$ & 7 (bueno) \\
\hline Gulati et al. 2014 & $S$ & $S$ & $\mathrm{~N}$ & $S$ & N & $\mathrm{N}$ & N & $S$ & $S$ & S & $S$ & 7 (bueno) \\
\hline $\begin{array}{l}\text { Nikpour et al. } \\
2014\end{array}$ & $S$ & $S$ & $\mathrm{~N}$ & $S$ & $S$ & S & S & $S$ & $\mathrm{~S}$ & $\mathrm{~S}$ & $\mathrm{~S}$ & $\begin{array}{l}10 \text { (muy } \\
\text { bueno) }\end{array}$ \\
\hline $\begin{array}{l}\text { Mashhood et al. } \\
2006\end{array}$ & $\mathrm{~s}$ & $\mathrm{~s}$ & $\mathrm{~N}$ & $\mathrm{~s}$ & $\mathrm{~s}$ & $\mathrm{~N}$ & $\mathrm{~N}$ & $\mathrm{~S}$ & $\mathrm{~s}$ & $\mathrm{~s}$ & $\mathrm{~s}$ & 8 (bueno) \\
\hline Jull et al. 2008 & $S$ & $\mathrm{~s}$ & $\mathrm{~N}$ & $\mathrm{~s}$ & $\mathrm{~s}$ & $\mathrm{~N}$ & $\mathrm{~N}$ & S & $S$ & S & $\mathrm{S}$ & 8 (bueno) \\
\hline Tsang et al. 2017 & S & S & $\mathrm{N}$ & S & $s$ & $\mathrm{~N}$ & $\mathrm{~N}$ & $S$ & $\mathrm{~S}$ & s & $S$ & 8 (bueno) \\
\hline $\begin{array}{l}\text { Gethin et al. } \\
2008\end{array}$ & $S$ & $S$ & $\mathrm{~N}$ & S & $\mathrm{N}$ & $\mathrm{N}$ & $\mathrm{N}$ & $S$ & $S$ & S & $S$ & 7 (bueno) \\
\hline $\begin{array}{l}\text { Gethin et al. } \\
2008\end{array}$ & $S$ & $\mathrm{~s}$ & $\mathrm{~N}$ & S & $\mathrm{S}$ & $\mathrm{s}$ & $\mathrm{N}$ & $S$ & $\mathrm{~s}$ & S & $\mathrm{S}$ & $\begin{array}{l}9 \text { (muy } \\
\text { bueno) }\end{array}$ \\
\hline $\begin{array}{l}\text { Shukrimi et al. } \\
2008\end{array}$ & $S$ & $S$ & $\mathrm{~N}$ & $S$ & $S$ & $\mathrm{~N}$ & $N$ & $S$ & $S$ & S & $S$ & 8 (bueno) \\
\hline $\begin{array}{l}\text { Agarwal et al. } \\
2015\end{array}$ & $S$ & $S$ & $\mathrm{~N}$ & $S$ & $N$ & $\mathrm{~N}$ & $\mathrm{~N}$ & $S$ & $S$ & S & $S$ & 6 (bueno) \\
\hline
\end{tabular}


$\begin{array}{lllllllllllllll}\text { Razzak et } & \text { al. } & \mathrm{S} & \mathrm{S} & \mathrm{N} & \mathrm{S} & \mathrm{N} & \mathrm{N} & \mathrm{N} & \mathrm{S} & \mathrm{S} & \mathrm{S} & \mathrm{S} & 6 \text { (bueno) }\end{array}$ 2005

$\begin{array}{lllllllllllll}\text { Lavandera } 2011 & \text { S } & S & S & S & N & N & N & N & S & S & N & 6 \text { (bueno) }\end{array}$

Tabla 3. Resultados escala AMSTAR.

\begin{tabular}{|c|c|c|c|c|c|c|c|c|c|c|c|c|}
\hline Autores & 1 & 2 & 3 & 4 & 5 & 6 & 7 & 8 & 9 & 10 & 11 & Puntuación \\
\hline $\begin{array}{l}\text { González et al. } \\
2004\end{array}$ & $S$ & $S$ & $S$ & $S$ & $S$ & $S$ & $N$ & $S$ & $\mathrm{~N}$ & $\mathrm{~N}$ & $N$ & 7 (bueno) \\
\hline $\begin{array}{l}\text { Rosanova et al. } \\
2012\end{array}$ & $S$ & $S$ & $S$ & $S$ & $S$ & $S$ & $S$ & $S$ & $\mathrm{~N}$ & $\mathrm{~N}$ & $N$ & 8 (bueno) \\
\hline Aziz et al. 2017 & $S$ & $S$ & $\mathrm{~S}$ & $\mathrm{~S}$ & $\mathrm{~S}$ & S & $\mathrm{S}$ & $\mathrm{S}$ & $\mathrm{N}$ & $S$ & $\mathrm{~N}$ & $\begin{array}{l}9 \text { (muy } \\
\text { bueno) }\end{array}$ \\
\hline $\begin{array}{l}\text { Wijesinghe et } \\
\text { al. } 2009\end{array}$ & $S$ & $S$ & $S$ & $\mathrm{~N}$ & $S$ & $S$ & $\mathrm{~N}$ & $S$ & $\mathrm{~N}$ & $\mathrm{~N}$ & $\mathrm{~N}$ & 6 (bueno) \\
\hline $\begin{array}{l}\text { Ventura et al. } \\
2017\end{array}$ & $S$ & $\mathrm{~N}$ & $N$ & $N$ & $S$ & $S$ & $\mathrm{~N}$ & $S$ & $\mathrm{~N}$ & $\mathrm{~N}$ & $N$ & 4 (justo) \\
\hline $\begin{array}{l}\text { Jason et al. } \\
2011\end{array}$ & $S$ & $S$ & $S$ & $N$ & $N$ & $N$ & $N$ & $S$ & $N$ & $N$ & $N$ & 4 (justo) \\
\hline $\begin{array}{l}\text { Justo et al. } \\
2015\end{array}$ & $S$ & $\mathrm{~N}$ & $S$ & $S$ & $S$ & $N$ & $\mathrm{~N}$ & $\mathrm{~N}$ & $\mathrm{~N}$ & $S$ & $\mathrm{~N}$ & 5 (justo) \\
\hline
\end{tabular}

VI. Análisis de los datos y niveles de evidencia.

Tabla 4. Niveles de evidencia.

$\begin{array}{ll}\text { Autores } & \begin{array}{l}\text { Nivel de } \\ \text { evidencia } \\ \text { científica }\end{array}\end{array}$




\begin{tabular}{|c|c|c|}
\hline $\begin{array}{l}\text { Baghel et al. } \\
2009\end{array}$ & $1+$ & ECA bajo riesgo de sesgo \\
\hline $\begin{array}{l}\text { Yapucu Günes U } \\
\text { et al. } 2007\end{array}$ & 1 & ECA con alto riesgo de sesgo \\
\hline $\begin{array}{l}\text { Kamaratos et al. } \\
2014\end{array}$ & $1++$ & ECA con muy bajo riesgo de sesgo \\
\hline Singh et al. 2011 & $1+$ & ECA con bajo riesgo de sesgo \\
\hline Ingle et al. 2006 & $1++$ & ECA con muy bajo riesgo de sesgo \\
\hline $\begin{array}{l}\text { Saeidinia et al. } \\
2017\end{array}$ & $1++$ & ECA con muy bajo riesgo de sesgo \\
\hline Imran et al. 2015 & $1+$ & ECA con bajo riesgo de sesgo \\
\hline Nurus et al. 2011 & $1+$ & ECA con bajo riesgo de sesgo \\
\hline Gulati et al. 2014 & $1+$ & ECA con bajo riesgo de sesgo \\
\hline $\begin{array}{l}\text { Nikpour et al. } \\
2014\end{array}$ & $1++$ & ECA con muy bajo riesgo de sesgo \\
\hline $\begin{array}{l}\text { Mashhood et al. } \\
2006\end{array}$ & $1+$ & ECA con bajo riesgo de sesgo \\
\hline Jull et al. 2008 & $1+$ & ECA con bajo riesgo de sesgo \\
\hline Tsang et al. 2017 & $1+$ & ECA con bajo riesgo de sesgo \\
\hline $\begin{array}{l}\text { Gethin et al. } \\
2008\end{array}$ & $1+$ & ECA con bajo riesgo de sesgo \\
\hline $\begin{array}{l}\text { Gethin et al. } \\
2008\end{array}$ & $1++$ & ECA con muy bajo riesgo de sesgo \\
\hline $\begin{array}{l}\text { Shukrimi et al. } \\
2008\end{array}$ & $1+$ & ECA con bajo riesgo de sesgo \\
\hline $\begin{array}{l}\text { Agarwal et al. } \\
2015\end{array}$ & $1+$ & ECA con bajo riesgo de sesgo \\
\hline $\begin{array}{l}\text { Razzak et al. } \\
2005\end{array}$ & $1+$ & ECA con bajo riesgo de sesgo \\
\hline $\begin{array}{l}\text { Rosanova et al. } \\
2012\end{array}$ & $2++$ & $\begin{array}{l}\text { RS de alta calidad de estudios de } \\
\text { cohortes }\end{array}$ \\
\hline Aziz et al. 2017 & $1+$ & RS de ECA bien hechas \\
\hline $\begin{array}{l}\text { Wijesinghe et al. } \\
2009\end{array}$ & 1 & Meta-análisis de ECA \\
\hline
\end{tabular}




\begin{tabular}{|l|l|l|}
\hline Lavandera 2011 & $1+$ & ECA con bajo riesgo de sesgo \\
\hline $\begin{array}{l}\text { González et al. } \\
2004\end{array}$ & $1+$ & RS con bajo riesgo de sesgo \\
\hline $\begin{array}{l}\text { Ventura et al. } \\
2017\end{array}$ & $2++$ & $\begin{array}{l}\text { RS de alta calidad de estudios de casos y } \\
\text { controles }\end{array}$ \\
\hline Jason et al. 2011 & $2++$ & $\begin{array}{l}\text { RS de alta calidad de estudios de casos y } \\
\text { controles }\end{array}$ \\
\hline Justo et al. 2015 & 1 & RS de ECA \\
\hline
\end{tabular}

Esta revisión consta de estudios con bajo riesgo de sesgo. En general, la evidencia, cuando se evalúa usando la escala SIGN, es de calidad normal-alta.

\section{Resultados}

Los resultados obtenidos ponen de manifiesto que la miel puede ser una alternativa de tratamiento eficaz con mejores resultados y más costo-efectivo que otros tratamientos tópicos.

Estos resultados se han agrupado en los siguientes epígrafes en función del objetivo de este trabajo en: tratamientos principales y otros tratamientos.

\section{Miel vs tratamientos principales.}

\section{Sulfadiazina de plata (SSD).}

Nueve estudios $(\underline{22-30})$ evaluaron el tratamiento en quemaduras de primer y segundo grado lavando con solución salina 0,9\% y aplicando sulfadiazina de plata o miel pura y vendando con gasas estériles.

Los estudios coinciden en señalar que el aderezo de miel causaba una cicatrización de la herida más rápida. En dos de los estudios $(\underline{22,23})$ las duraciones promedio de curación de los pacientes en el grupo de miel fue 18 días y en el 
grupo SSD 32 días, también se disminuía el dolor y las heridas se volvían estériles en menos tiempo como se muestra en la figura 1, mientras que en un tercer estudio ${ }^{(24)}$ el tiempo de curación fue de 15 días en el grupo de la miel y de 20 días para SSD.

Tabla 5. Elaboración propia.

\begin{tabular}{|l|l|l|}
\hline \multicolumn{1}{|c|}{ Tiempo de esterilidad } & Miel & \multicolumn{1}{|c|}{ SSD } \\
\hline 7 días & $62 \%$ & $0 \%$ \\
\hline 14 días & $99,5 \%$ & $14,2 \%$ \\
\hline 21 días & $100 \%$ & $42,8 \%$ \\
\hline
\end{tabular}

Respecto a la esterilidad, en el estudio de Razzak et al. (24), al tomar hisopos de las heridas se reveló que los pacientes tratados con miel no mostraron crecimiento de ningún organismo patógeno, pero el $80 \%$ de los pacientes del otro grupo mostró crecimiento de diferentes patógenos durante su tratamiento, necesitando el uso de terapia sistémica con antibióticos en consecuencia.

Asimismo, otros autores juzgaron los resultados en base a tres criterios. Estos incluyeron la velocidad de curación de heridas, la rapidez de alivio del dolor sin medicamentos orales y el tiempo que tarda la herida en esterilizarse. El grupo I tratado con miel el $100 \%$ de los pacientes se curaron a las 4 semanas, no tuvieron dolor y las heridas se volvieron estériles a las 3 semanas, por el contrario en el grupo II tratado con sulfadiazina de plata al 1\%, el 100\% fueron curados al final de las 6 semanas del tratamiento, no tenían dolor a las 4 semanas y se volvieron estériles a las 5 semanas $(\underline{25}, \underline{26})$ (tabla 6 ).

Tabla 6. Elaboración propia.

Tiempo de: $\quad$ Miel $\quad$ SSD




\begin{tabular}{|l|l|l|}
\hline Curación & 4 semanas & 6 semanas \\
\hline Dolor & 3 semanas & 4 semanas \\
\hline Esterilidad & 3 semanas & 5 semanas \\
\hline
\end{tabular}

Estos ensayos también revelaron una generación de cicatriz hipertrófica, que fue un $4 \%$ mayor en el grupo de SSD en comparación con el grupo tratado con miel durante el seguimiento. Además los pacientes se quejaron de prurito en el área curada y pigmentación/hipopigmentación en el área cicatrizada, siendo la mayoría de las quejas del grupo de sulfadiazina de plata mientras que sólo una pequeña parte provenía del grupo de miel (13 vs 2 respectivamente). De igual modo, no hubo reacciones alérgicas ni efectos secundarios en el grupo de miel; sin embargo, si se observó una leve irritación de 2 pacientes en el grupo de sulfadiazina argéntica. Por consiguiente, las heridas tratadas con miel tienen un mejor resultado en términos de cicatrización hipertrófica y contracturas posteriores a la quemadura (menos cicatrices) comparado con la SSD. Además de no haber efectos secundarios como alergia, irritación o toxicidad (22-2흐).

Respecto al costo del tratamiento, varios autores mostraron que el tratamiento con miel fue 10 veces más bajo que el SSD (1\%) y como la duración de la curación de heridas con miel era menor, se requirió un menor número de apósitos en el grupo I en comparación con el grupo II, lo que redujo aún más el costo del tratamiento con miel (24-26).

\section{Povidona iodada.}

Tres estudios $(\underline{31}-\underline{33})$ evaluaron la miel pura sin procesar comparado con povidona iodada en úlceras grado II de pie diabético. Las heridas se limpiaron inicialmente con suero salino $0,9 \%$, se aplicó miel o povidona iodada y se cubrieron con gasas que fueron cambiadas diariamente. El tiempo de curación osciló entre 14 días y 6 semanas. 
En dos de los estudios (32,33), las heridas en el grupo de povidona iodada tomaron una duración media de 15 días para el cierre, mientras que en el grupo de miel las heridas requieren una duración media de 14 días, por lo que la diferencia en la duración no fue estadísticamente significativa. Por el contrario, en el estudio de Gulati et al. ${ }^{(31)}$ el porcentaje de sanación completa en el grupo tratado con miel fue de $31.82 \%$, y ninguno de los sujetos logró la curación completa con povidona yodada en el mismo periodo. Se produjo una reducción del área de superficie de las heridas de 4,35 cm2 a 0,55 cm2 en el grupo de la miel y de $4,25 \mathrm{~cm} 2$ a $1,95 \mathrm{~cm} 2$ en el grupo de povidona de yodo a las 6 semanas.

Respecto al dolor se analizó mediante la escala analógica visual (EVA). La media de puntuación al inicio fue de $7 / 10$ y se redujo a $1 / 10$ al final del tratamiento para el grupo de la miel. Por otra parte, en los pacientes del grupo povidona yodada el puntaje de dolor pasó de 7/10, al inicio del estudio, a 5/10. También puntuaron el estado de comodidad general que fue mayor para los pacientes tratados con miel. Todos los pacientes de este último grupo experimentaron menos dolor durante el vendaje, además de resolverse antes el edema y los exudados malolientes en comparación con el grupo de povidona yodada $(\underline{31}-\underline{33})$.

Otro ensayo (34) realizado en pacientes con heridas postoperatorias infectadas (cesáreas), reveló que el $84,4 \%$ de los pacientes tratados con miel se curó completamente mientras en el grupo tratado con povidona iodada la curación sólo se produjo en el 50\% y habiendo en este último un mayor número de dehiscencias y necesidad de cierre de la herida por segunda intención.

Estos resultados ponen de manifiesto que la miel produce menos dolor y disminuye el edema y el olor de la herida de manera más efectiva $\left.{ }^{(31}-34\right)$.

\section{Antibióticos locales.}

Tres estudios (34-36) evaluaron la efectividad de la miel comparándola con antibióticos locales (gentamicina, nitrofurazona, neomicina y facdermin) en heridas sépticas; se hizo una cura local diaria con solución salina al 0,9 \% y posterior aplicación de antibióticos locales o de miel de abeja.. Se observó que 
la miel producía tejido de granulación y cierre de la herida en menor tiempo, eliminaba más rápido el olor característico de las lesiones y el tejido desvitalizado, el enrojecimiento, el edema perilesional y las secreciones purulentas, haciendo la herida estéril en menos tiempo; no se observaron efectos adversos y se ahorraron 40,75 € por paciente comparado con los antibióticos, concluyendo con un alto grado de efectividad y coste-efectividad de la miel respecto a los antibióticos tópicos convencionales.

Un cuarto estudio ( ${ }^{(37)}$ evaluó la eficacia de la miel frente a nitrofurazona y etoxi-diaminoacridina en úlceras grado II y III. Se observó que en la 5ª semana de tratamiento la tasa de curación fue 4 veces mayor en la miel con respecto al otro. Además, no se registró ningún efecto adverso del tratamiento con miel.

Tratamientos convencionales (solución salina, gasas mojadas, vendajes).

Cinco estudios $(\underline{32,38-41)}$ evaluaron la miel en diferentes tipos de úlceras (neuropáticas, venosas, mixtas y UPP) comparado con tratamientos convencionales (solución salina, gasas mojadas y vendajes). En los estudios se observó una mayor cicatrización de las heridas y una rápida esterilización en el grupo de la miel respecto a los tratamientos convencionales, los cuales precisaron antibioterapia e incluso en algunos casos hospitalización, concluyendo que la miel presenta una curación acelerada además de tener un efecto antibacteriano.

Respecto a la curación, la duración promedio de la curación fue de 31 días en el grupo tratado con miel comparado con 43 días en el grupo tratado con apósitos convencionales ${ }^{(38)}$. Sin embargo, en un segundo estudio (모) el tiempo medio de curación de la herida fue de 18 días en el grupo tratado con miel y 29 días en el grupo de apósitos convencionales. No se observaron efectos secundarios graves en ambos grupos $(\underline{38}-\underline{39})$. Sin embargo, otros estudios $(\underline{42}, 4 \underline{4})$ observaron que la miel no presenta diferencia estadística en la curación comparado con los tratamientos convencionales en úlceras en piernas. 
Respecto a la esterilidad, los resultados revelaron que el $100 \%$ de las heridas fueron estériles a las 4 semanas en el grupo de la miel y a las 6 semanas en el otro grupo. Además, este último requirió antibioterapia y en algunos casos hospitalización (38-40).

Así mismo, se realizó un seguimiento de varios ítems ${ }^{(40)}$ que se muestran en la tabla 7:

Tabla 7. Elaboración propia.

\begin{tabular}{|l|l|l|}
\hline İtems & Grupo miel & Grupo ap. convencional \\
\hline \% sanación & $55,6 \%$ & $49,7 \%$ \\
\hline Tiempo curación & 63,5 días & 65 días \\
\hline Reducción área úlcera & $74 \%$ & $65,5 \%$ \\
\hline Infección & $17,1 \%$ & $22,1 \%$ \\
\hline Rentabilidad & 13.euros & 32 euros \\
\hline
\end{tabular}

\section{Miel vs otros tratamientos tópicos.}

\section{Hidrogeles.}

En cuanto a la miel comparada con hidrogeles en úlceras venosas crónicas y heridas superficiales y abrasiones, se observó que la miel, previa aplicación de suero salino 0,9\% para limpiar las heridas, demostró una mayor incidencia de curación, desbridamiento eficaz y menos tasa de infección, además de ser más eficaz para erradicar SARM (Staphylococcus aureus resistente a la meticilina), respecto a los hidrogeles ${ }^{(42-45)}$ y, aunque otros autores afirmaron que no existe gran diferencia entre la miel y el intraSite Gel, sí evidenciaron ser más coste-efectiva (46).

\section{Placebo.}


En cuanto a este efecto, se evaluó un gel de miel en heridas quirúrgicas por cesárea comparado con placebo. El gel estaba formado en mayor proporción por miel ( $25 \mathrm{gr}$ ) y agua destilada $(69.3 \mathrm{gr}$ ) y en menor cantidad por glicerina ( 5 $\mathrm{gr})$, carbopol $(0,55 \mathrm{gr})$, trietanolamina $(0,5 \mathrm{gr})$, metil parabeno $(0,18 \mathrm{gr})$ y propil parabeno $(0,02 \mathrm{gr})$; por otro lado el placebo estaba formado por los mismos compuestos y en las mismas cantidades pero carecía de miel. Se observó que no hubo diferencias significativas para ambos grupos respecto al consumo de antibióticos, alimentación y actividad habitual pero la tasa de curación de la miel fue mayor respecto al grupo placebo ( $86 \%$ y $26 \%$ respectivamente) $(\underline{47})$.

\section{Clorhexidina al $0,05 \%$.}

También se ha estudiado la aplicación de la miel en heridas postoperatorias infectadas sin respuesta al tratamiento convencional. Se observó que el uso de antibioterapia sistémica y limpieza de la herida con clorhexidina al 0,05\% durante 14 días o más no daba resultado. La aplicación tópica de miel sin procesar dio como resultado una marcada mejoría clínica a los 5 días y una resolución del $100 \%$ de las heridas a los 21 días, sin ninguna reacción adversa local (36).

\section{Apósito de Plata Nanocristalina (nAg).}

En cuanto a la comparativa de la miel con apósitos de plata nanocristalina (nAg) en úlceras de pie diabético, se observó que la curación del apósito de plata es potencialmente mejor en términos de reducción de tamaño de la úlcera en comparación con la miel, aunque se encontraron limitaciones por el pequeño tamaño de la muestra y el corto tiempo de evaluación (42).

\section{Discusión}


Los resultados obtenidos en esta revisión sistemática para valorar la evidencia científica sobre la efectividad de la miel como un tratamiento alternativo efectivo en quemaduras, heridas o úlceras manifiestan en términos generales que la miel es potencialmente más efectiva necesitando menos tiempo para cicatrizar y lograr mayor esterilidad, además de producir menos dolor, reducir el edema y las secreciones malolientes, confiriendo mayor esterilidad a las lesiones en un menor tiempo (siendo efectiva incluso contra las cepas resistentes a los antibióticos), con menos efectos adversos y más costo-efectiva comparado con otros tratamientos, por lo que el objetivo explicitado en la pregunta PICO se cumple.

Se ha comprobado que el aderezo de miel disminuye el tiempo de curación completa de las lesiones, produciendo una cicatrización más rápida de las heridas, sin embargo, algunos autores refieren que el tiempo de cicatrización no es estadísticamente significativo en comparación con los otros tratamientos. A pesar de ello, ésta posee otras ventajas durante el proceso de curación respecto a dichos tratamientos debido a sus propiedades. Todo ello ayuda a reducir la incidencia de infección, la cual es una de las complicaciones más frecuentes de la cicatrización y representa una considerable morbilidad, malestar y hospitalización prolongada.

En relación con las repercusiones emocionales que este tipo de lesiones puede generar, destaca el temor relacionado al dolor y evolución de la herida, y el daño a la imagen corporal que éstas producen. En ocasiones, las lesiones requieren tratamientos invasivos y prolongados que pueden conllevar complicaciones y contribuir a la aparición de alteraciones psicológicas debido al dolor que provocan. Diversos estudios evaluaron las alteraciones emocionales en pacientes con quemaduras hospitalizados y en sus familiares y evidenciaron cuadros de ansiedad, emoción de miedo y alteraciones del sueño, asociados al dolor producido por las lesiones y las curas.

Del mismo modo, las cicatrices que quedan tras estas heridas pueden cambiar la manera en que la persona percibe su cuerpo, pudiendo originar un trastorno de la imagen corporal. Esta alteración suele estar asociada con una baja autoestima que desemboca en estados ansiedad y depresión, 
acompañados de aislamiento social y problemas sexuales. Debido a que la percepción del propio cuerpo se ve influenciada por factores sociales, la persona con una alteración sobre su imagen puede sentirse incómoda al interaccionar con otras personas, pudiendo pensar que sus cicatrices son desagradables y preocupándose de que los demás los rechacen por éstas. Sobre todo, en los niños, éstas pueden ser objeto de burlas que puede llevar a caer en depresión o sufrir ansiedad cuando conocen a gente nueva.

Por consiguiente, puesto que uno de los mayores problemas con los que se encuentra el profesional de enfermería es la cura satisfactoria de heridas, siendo en algunas ocasiones complicado dar con el tratamiento adecuado, la miel debería contemplarse como tratamiento alternativo en este tipo de lesiones.

\section{Conclusiones}

Los resultados obtenidos pueden conducir a las siguientes conclusiones:

- La miel puede ofrecerse como alternativa en el tratamiento de quemaduras, heridas y úlceras debido a su beneficiosa curación.

- Mejora el resultado estético de la herida dejando menos cicatriz.

- Disminuye el impacto sobre la imagen corporal de este tipo de lesiones.

- Supone un tratamiento más barato y eficaz.

Pudiendo suponer una mejora de la calidad en la asistencia sanitaria, reducir el número de procedimientos innecesarios, disminuir la variabilidad clínica y unificar los criterios de actuación entre los profesionales. 


\section{Bibliografía}

1. Viñas Díaz JA, Juan Rodríguez J, González Rodríguez M. Epidemiología de las lesiones por quemaduras. Sociedades científicas. 2009 Abril 6 : 1-8.

2. Solís F, Domic C, Saavedra R, González A. Incidencia y prevalencia de las lesiones por quemaduras en menores de 20 años. Rev Chil Pediatri. 2014; 85(6): 674-681.

3. Mock C, Peck M, Peden M, Krug E, eds. A WHO plan for burn prevention and care. World Health Organization. 2008

4. Baltà Domínguez I, me Valls Colomé, M. Quemaduras. Actualización en Medicina de Familia. AMF. 2011; 7(10): 584-590

5. Santalla A, López-Criado MS, Ruiz MD, Fernández-Parra J. Infección de la herida quirúrgica. Prevención y tratamiento. Clin Invest Gin Obst. 2007; 34(5): 189-96.

6. Sociedad Española de Medicina Preventiva, Salud Pública e Higiene. Estudio de Prevalencia de las infecciones nosocomiales en España. Estudio EPINE-EPPS 2016. Resultados provisionales;consultado Feb;. Disponible en: http://hws.vhebron.net/epine/Global/EPINEEPPS\%202016\%20Informe\%20Global\%20de\%20Espa\%C3\%B1a\%20Resu men.pdf

7. Blanco López JL. Definición y clasificación de las úlceras por presión. El Peu. 2003; 23(4): 194-198.

8. Pancorbo-Hidalgo PL, García-Fernández FP, Torra Bou JE, Verdú Soriano J, Soldevilla-Agreda JJ. Epidemiología de las úlceras por presión en España en 2013: 4º Estudio Nacional de Prevalencia. Gerokomos. 2014; 25(4): 162170.

9. Real López L, Diez Estébanez ME, Serrano Hemantes M, de la Iglesia García E, Blasco Romeo I, Capa Santamaría S, et al. Prevalencia de úlceras 
por presión y lesiones curáneas a la humedad en el Hospital Universitario de Burgos. Gerokomos. 2017; 28(2): 103-108

10. Singh N, Amstrong DG, Lipsky BA. Preventing Foot Ulcers in Patients with Diabetes. JAMA. 2005: 217-228.

11. Guila Fidel S, Fernández MJ, Argüello JM. Aspectos emocionales de las personas con quemaduras. Kreamics.

12. Johnson K, Krause J. La ansiedad psicológica después de una lesión por quemadura. MSKTC. 2011.

13. Fernández-Vega Barreto FL, Puebla Farigola I, Carrillo Vázquez L. Alteraciones psicológicas en pacientes ingresados por quemadura. Multimed. 2015; 19 (1).

14. Cabello Jaime R, Del Cubo Arroyo J, Gálvez Esquinas MJ, García Díaz FJ, Martín Montañez E, Molina Alonso JM, et al. Manejo del paciente con deterioro de la integridad cutánea. Conserjería de salud. 2007: 132-136

15. Jiménez García JF, Barroso Vázquez M, DE Haro Fernández F, Hernández López MT. Guía de práctica clínica para la prevención y cuidados de las úlceras arteriales. Servicio Andaluz de Salud. 2009: 37-41

16. Jull AB, Cullum N, Dumville JC, Westby MJ, Deshpande S, Walker N. Honey as a topical treatmente for wounds. The Cochrane Library Internet. 2015 Mar citado 17 Feb 2018 6(3). Disponible en: http://cochranelibrarywiley.com/doi/10.1002/14651858.CD005083.pub4/epdf

17. Mengarelli $\mathrm{RH}$, Bilevich E, Belatti A, Gorosito S. Agentes tópicos tradicionales utilizados para la cura de heridas ¿mito o verdad?. Act Terap Dermatol. 2013; 36: 101

18. Bitter CC, Erickson TB. Management of Burn Injuries in the Wilderness: Lessons from Low-Resource Setting. Wilderness \& environmental medicine. 2016; 27: 519-525. 
19. Schencke C, Vásquez B, Sandoval C, Del Sol M. El rol de la miel en los procesos morfofisiológicos de reparación de heridas. Int J Morphol. 2016; 34: 385-395.

20. Molan PC. The evidence and the rationale for the use of honey as a wound dressing. Wound practice and research. 2011; 19(4): 204- 220.

21. Haynes JS, Callaghan R. Properties of honey: its mode of action and clinical outcomes. Wounds UK. 2011; 7(1): 50-57.

22. Baghel PS, Shukla S, Mathur RK, Randa R. A comparative study to evaluate the effect of honey dressing and silver sulfadiazene dressing on wound healing in burn patients. Indian J Plast Surg. 2009; 42(2): 176-181

23. Singh Gupta S, Singh O, Bhagel PS, Moses S, Shukla S, Mathur RK. Honey dressing versus silver sulfadiazene dressing for wound healing in burn patients: a retrospective study. Journal of Cutaneous and Aesthetic Surgery. 2011; 4(3).

24. Razzak Memon A, Tahir SM, Khushk IA, Ali Memon G. Therapeutic effects of honey versus silver sulfadizine in the management of burn injuries. JLUMHS. 2005.

15. Nurus Sami A, Mehmood N, Azhar Qureshi M, Zeeshan HK, Malik Irfan A, lqbal Khan M. Honey compared with silver sulphadiazine as burn wound dressing. Ann. Oak. Inst. Med. Sci. 2011; 7(1): 22-25.

26. Mashhood AA, Khan TA, Sami AN. Honey compared with $1 \%$ silver sulfadiazine cream in the treatment of superficial and partial thickness burns. Journal of Pakisan Association of Dermatologist. 2006; 16: 14-19.

27. Saeidinia A, Keihanian F, Lashkari AP, Ghawami Lahiji H, Mobayyen M, Heidarzade $A$, et al. Partial-thickness burn wounds healing by topical treatment. Medicine. 2017; 96(9).

28. Wijesinghe M, Weatherall M, Perrin K, Beasley R. Honey in the treatment of burns: a systematic review and meta-analysis of its efficacy. New Zealand Medical Journal. 2009; 122(1295): 47-60. 
29. Aziz Z, Rasool Hassan BA. The effects of honey compared to silver sulfadiazine for the treatment of burns: a systematic review of randomized controlled trials. Burns. 2017; 43: 50-57.

30. Rosanova MT, Stamboulian D, Lede R. Revisión sistemática: ¿Cuál es el agente tópico más eficaz en la prevención de infecciones en el paciente quemado?. Arch Argent Pediatric. 2012; 110(4): 298-303.

31. Gulati S, Qureshi A, Srivastava A, Kataria K, Kumar P, Ji AB. A pospective randomized study to compare the effectiveness of honey dressing vs povidone iodine dressing in chronic wound healing. Indian J Surg. 2014; 76(3): 193-198.

32. Shukrimi A, Sulaiman AR, Halim AY, Azril A. A comparative study between honey and povidone iodine as dressing solution for Wagner Type II diabetic foot ulcers. Med J Malaysia. 2008; 63.

33. Agarwal S, Bhardwaj V, Singh A, Khan H, Goel S, Bharat M, et al. A control clinical trial of honey-impregnated and povidone iodine dressing in the treatment of diabetic foot ulcers among northern indian subjects. Indian J Sci Res. 2015; 6(2): 7-10.

34. Ventura Rodríguez DP. Efectividad del uso de la sacarosa en el proceso de cicatrización de heridas. trabajo fin de máster; Vega de San Mateo: Universidad Miguel Hernández. 2017. citado 10 de enero de 2018. Recuperado a partir de: http://dspace.umh.es/bitstream/11000/4439/1/VENTURA\%20RODRIGUEZ\%2C \%20DAVINIA\%20DEL\%20PINO.pdf

35. Lavandera Rodríguez I. Curación de heridas sépticas con miel de abejas. Revista Cubana de Cirugía. 2011; 50(2):187-196.

36. González Gascón R, Del Dedo Torre P. Actualización sobre el uso de miel en el tratamiento de úlceras y heridas. Caso clínico. Enfermería global. 2004.

37. Yapucu Günes U, Eser I. Effectiveness of a honey dressing for healing pressure ulcers. Journal of wound, ostomy and continence nursing. 2007; 34(2): 184-190. 
38. Kamaratos AV, Tzirogiannis KN, Iraklianou SA, Panoutsopoulos GI, Kanellos IE, Melidonis Al. Manuka Honey-impregnated dressing in the treatment of neuropathic diabetic foot ulcers. Int Wound J. 2014; 11: 259-263.

39. Imran M, Hussain MB, Baig M. A randomized controlled clinical trial of honey-impregnated dressing for trating diabetic foot ulcer. Journal of the College of Physicians and Surgeon Pakistan. 2015; 25(10): 721-725.

40. Jull A, Walker N, Parag V, Molan P, Rodgerns A. Randomized clinical trial of honey-impregnated dressing for venous leg ulcers. British Journal of Surgery. 2008; 95: 175-182

41. Tsang KK, Wai-yung Kwong E, Shing-shun To T, Wai-Yee Chung J, KwokShing Wong T. A pilot randomized controlled study of nanocrystalline silver, manuka honey, and conventional dressing in healing diabetic foot ulcer. Evidence-Based Complementary and Alternative Medicine. 2017.

42. Jason J, Song MD. Use of honey in wound care. Adv skin wound care. 2011; 24: 40-44.

43. Justo Rubio E, Guerra Cuesta I. Eficacia de la miel y apósitos de miel en UPP. trabajo fin de grado Valladolid Universidad de Valladolid. 2015. citado 1 de Febrero de 2018. Recuperado a partir de: https://uvadoc.uva.es/bitstream/10324/11873/1/TFG-H247.pdf

44. Gethin G, Cowman S. Bacteriological changes in sloughy venous leg ulcers treated with manuka honey or hidrogel: An RCT. Journal of Wound Care. 2008; 17(6).

45. Gethin G, Cowman S. Manuka honey vs. hydrogel - a prospective, open label, multicentre, randomised controlled trial to compare desloughing efficacy and healing outcomes in venous ulcers. Journal of Clinical Nursing. 2008.

46. Ingle R, Levin J, Polinder K. Wound healing with honey-a randomised controlled trial. SAMJ. 2006; 96(9):831-835

47 Nikpour M, Shirvani MA, Azadbakht M, Zanjani R, Mousavi E. The effect of honey gel on abdominal wound healing in cesarean section: a triple blind randomized clinical trial. Oman Medical Journal. 2014; 29(4): 255-259 
LA MIEL COMO ALTERNATIVA A LOS TRATAMIENTOS TÓPICOS EN EL PROCESO DE CURACIÓN DE QUEMADURAS, HERIDAS Y ÚLCERAS 